\title{
Over-Expressions of Serum miR-182-5p, miR-363-3p, and miR-378a-3p serve as Biomarkers in Hepatocellular Carcinoma
}

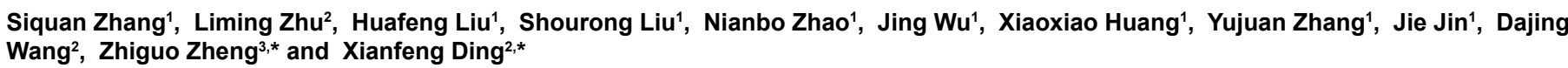

${ }^{1}$ Xixi Hospital of Hang Zhou, Hangzhou 310023, China

${ }^{2}$ College of Life Sciences, Zhejiang Sci-Tech University, Xiasha 2\# road,Hangzhou,Zhejiang 310018, PR China

${ }^{3}$ Zhejiang Cancer Research Institute, Zhejiang Cancer Hospital, Hangzhou 310022, China

\begin{abstract}
Serum a-fetoprotein (AFP) has been used as an early diagnostic biomarker of hepatocellular carcinoma (HCC), however the sensitivity and specificity of detection are poor. Therefore, new markers remain to be explored. MicroRNAs (miRNAs) are a class of small RNAs which could contribute to tumorigenesis by interaction with targeted mRNAs. MiRNAs are abundant in the circulating blood and are potentially useful for early diagnosis of hepatocellular carcinoma. In this study, we screened a group of candidate miRNAs (miR-182-5p, miR-363-3p, and miR-378a-3p) in serum, which were found to be up-regulated in HCC patients compared with in the healthy controls. Analysis of the receiver operating characteristic $(\mathrm{ROC})$ curve suggested that these three serum miRNAs had merits in the diagnosis of HCC (AUC $=0.802,0.845,0.880$, respectively). Furthermore, these candidate miRNAs can also differentiate HCC patients with negative AFP level from the healthy controls ( $A U C=0.865,0.930,0.970$, respectively). In conclusion, our results suggested that serum miR-182-5p, miR-363-3p, and miR-378a-3p might be applied as potential non-invasive biomarkers for HCC diagnosis, which is especially important for AFP-negative patients.
\end{abstract}

Keywords: MicroRNA; Hepatocellular carcinoma; AFP-Negative biomarker

\section{Introduction}

Hepatocellular carcinoma (HCC) is one of the most common cancers and represents a leading cause of malignancy-related death, with approximately 750,000 new cases and about 700,000 deaths annually worldwide [1]. Due to the lack of effective early detection or therapeutic strategy, the majority of HCC patients are diagnosed when they reach medium or even terminal stage, which leads to high mortality [2]. At present, surgical resection or liver transplantation are the main therapeutic methods to increase survival for HCC patients. The overall 5 -year survival rate for liver cancer is about $14 \%$ worldwide [1]. In clinical diagnosis for primary HCC, $\alpha$-fetoprotein level is a main indicator. However, its sensitivity and specificity have been far from satisfactory [3]. Approximately $20 \%$ of HCC patients with low AFP level are poorly diagnosed [4]. Therefore, screening potential biomarkers to effectively detect early tumor development is an important step towards HCC diagnosis and mortality reduction [5]. MicroRNAs are a class of endogenous, noncoding RNAs with $\sim 22$ nucleotides in length that are cleaved from 70- to 100-nt hairpin-shaped precursors [6]. MiRNAs regulate gene expression at the post-transcriptional level by binding partial sequencesto the 3 ' untranslated region (3'UTR) of target mRNAs. The binding interaction causes translational inhibition and/or mRNA degradation [7]. Moreover, dysregulation of miRNAs expression may contribute to tumorigenesis by inhibiting the expression of tumor suppressor genes or promoting the expression of proto-oncogenes [8]. Several studies have demonstrated that miRNAs have high stability in human serum or plasma due to protection from RNases [9-11], suggesting their potential use as diagnostic biomarkers. Therefore, profiling miRNA expression pattern may help to clarify the biological functions of miRNAs in hepatocarcinogenesis [6], which has considerable clinical value.

\section{Materials and Method}

\section{Patient characteristics and clinical features}

This study was approved by the Institutional Review Board of Zhejiang Sci-Tech University and Hangzhou Xixi Hospital (Hangzhou, China). Written informed consent was obtained from each participant.
Furthermore, this study was conducted in accordance with ethical statement in the Declaration of Helsinki by the World Medical Association. A total of 77 participants including 52 HCC patients and 25 normal subjects were recruited into this study. The clinical pathological characteristics of the participants are summarized in Table 1.

\section{RNA isolation from human serum samples}

One milliliter of venous whole blood was drawn from participants, allowed to clot for $30 \mathrm{~min}$ at room temperature, and then centrifuged at low speed for $10 \mathrm{~min}$. The supernatant serum was collected and preserved at $-80^{\circ} \mathrm{C}$. According to the manufacturer's protocol (Qiagen miRNeasy Mini Kit, Qiagen, Hilden, Germany), $200 \mu \mathrm{l}$ of serum was used for total RNA extraction. Total RNA containing miRNA was eluted with $50 \mu \mathrm{l}$ of the Qiagen RNase-free water. RNA concentrations were assessed with a NanoDrop ND-2000 spectrophotometer (NanoDrop Technologies, Wilmington,USA). Concentration of serum RNA ranged from 8.8 to $17.8 \mathrm{ng} / \mu \mathrm{l}$.

\section{Solexa sequencing and validation}

For the initial analysis of miRNA profile in HCC, the study was divided into two phases. The first phase of this study was designed to screen differential expression of miRNAs between HCC patients and healthy controls. Sample pooling was considered as a powerful, costeffective, and rapid means to identify the changes in a gene expression

*Corresponding authors: College of Life Sciences, Zhejiang Sci-Tech University, Xiasha 2\# road,Hangzhou ,Zhejiang 310018, PR China, Tel: 0571-86843516; Cellphone: 86-136-1581-0087; Fax: +86-571-86843303; E-mail: xfding@zstu.edu.cn

Zhiguo Zheng, Zhejiang Cancer Research Institute, Zhejiang Cancer Hospital Hangzhou 310022, China, Tel: +86-571-88122421; E-mail: zhengzg@zjcc.org.cn

Received December 09, 2015; Accepted January 18, 2016; Published January 25,2016

Citation: Zhang S, Zhu L, Ding X, Zhu C, Zheng Z (2016) Over-Expressions of Serum miR-182-5p, miR-363-3p, and miR-378a-3p serve as Biomarkers in Hepatocellular Carcinoma. Mol Biol 5: 154. doi:10.4172/2168-9547.1000154

Copyright: $\odot 2016$ Zhang S, et al. This is an open-access article distributed under the terms of the Creative Commons Attribution License, which permits unrestricted use, distribution, and reproduction in any medium, provided the original author and source are credited. 
Citation: Zhang S, Zhu L, Ding X, Zhu C, Zheng Z (2016) Over-Expressions of Serum miR-182-5p, miR-363-3p, and miR-378a-3p serve as Biomarkers in Hepatocellular Carcinoma. Mol Biol 5: 154. doi:10.4172/2168-9547.1000154

Page 2 of 7

\begin{tabular}{|c|c|c|c|c|}
\hline Parameters & $\begin{array}{c}\text { Sequencing set Healthy } \\
\text { Control }(n=5)\end{array}$ & $\begin{array}{l}\text { HCC patients } \\
(n=5)\end{array}$ & $\begin{array}{c}\text { Validation set } \\
\text { Healthy Control }(n=25)\end{array}$ & $\begin{array}{l}\text { HCC patients } \\
(n=52)\end{array}$ \\
\hline Age, median, years & 33 & 51 & 29 & 57 \\
\hline Men, $n(\%)$ & $3,60.0 \%$ & $4,80.0 \%$ & $19,76.0 \%$ & $40,76.9 \%$ \\
\hline \multicolumn{5}{|l|}{ AFP (ng/ml) } \\
\hline$\leq \mathbf{2 0}$ & 5 & 1 & 25 & 16 \\
\hline$>20$ & & 4 & & 36 \\
\hline \multicolumn{5}{|c|}{ Hepatitis B virus DNA copies (IU/ml) } \\
\hline$\leq 1000$ & 5 & 2 & 25 & 20 \\
\hline$>1000$ & & 3 & & 32 \\
\hline \multicolumn{5}{|l|}{ HBsAg level } \\
\hline negative & 5 & 0 & 25 & 2 \\
\hline positive & & 5 & & 50 \\
\hline \multicolumn{5}{|l|}{ Alanine aminotransferase (U/I) } \\
\hline$\leq 40$ & 5 & 1 & 25 & 29 \\
\hline$>40$ & & 4 & & 23 \\
\hline \multicolumn{5}{|c|}{ Aspartate aminotransferase (U/I) } \\
\hline$\leq \mathbf{4 0}$ & 5 & 1 & 25 & 20 \\
\hline$>40$ & - & 4 & & 32 \\
\hline Intrahepatic metastasis & - & 1 & - & 17 \\
\hline Distant metastasis & - & 0 & - & 6 \\
\hline Portal vein tumor thrombus & - & 0 & - & 12 \\
\hline \multicolumn{5}{|l|}{ Tumor size } \\
\hline$\leq 5$ & - & 4 & - & 28 \\
\hline$>5$ & - & 1 & - & 24 \\
\hline \multicolumn{5}{|l|}{ TNM phase } \\
\hline I phase & - & 3 & - & 13 \\
\hline Il phase & - & 1 & - & 15 \\
\hline III phase & - & 1 & - & 20 \\
\hline IV phase & - & 0 & - & 4 \\
\hline
\end{tabular}

Table 1: Summary of clinical details of subjects.

profile [12]. For Solexa sequencing, 5 HCC patients serum were pooled together and 5 healthy control samples were pooled together, respectively, to reduce the individual difference [13]. Small RNA molecules with the length of less than 50 bases were ligated with a pair of Solexa adaptors, reverse transcribed and amplified. The cDNA library was directly sequenced using Illumina's Solexa Hiseq 2000 Sequencer (Illumina, San Diego, USA). Finally, clean reads were compared with the miRBase database (version 20.0), and the abundance value of each known miRNA was normalized using "transcripts per million (TPM)" in both samples [14]. The differential expression of miRNA between two groups was revealed by clustering analysis. To validate this result, all samples (52 HCC and 25 controls) were further studied for the expression levels of interested miRNAs by real-time quantitative polymerase chain reaction (qRT-PCR) assay. qRT- PCR assay Serum RNA (2.5 $\mu \mathrm{l})$ from each sample was used in reverse transcription reaction, using $2 \mathrm{mM}$ miRNA-specific stem-loop primers and M-MLV reverse transcriptase according to the instruction of the manufacturer (Takara, Dalian, China). Primers for miR-182-5p, miR-363-3p, miR-378a-3p and RNU6 were designed by Primer premier 5 software (Premier Inc., Ontario Ottawa, Canada) and the sequences were listed in Supplementary Table S1. qRT-PCR was performed with ABI Prism 7500 RT-PCR system (Applied Biosystems, Foster City, USA), using Takara SYBR ${ }^{\circledR}$ Premix Ex Taq $^{\text {Tit }}$ II (Tli RNaseH Plus) (Takara). Each amplification reaction was conducted in a total volume of $20 \mu \mathrm{l}$, containing $1 \mu \mathrm{l}$ cDNA ( 10 ng/ reaction), $10 \mu \mathrm{l}$ 2X SYBR Green PCR Master mix, $0.4 \mu \mathrm{l}$ 50X ROX, 0.8 $\mu \mathrm{l} 10 \mathrm{mM}$ primer mix, and $7.8 \mu \mathrm{l}$ RNase-free water. Every sample was amplified in triplicates. The expression of the miRNAs in the HCC cases and the healthy controls was normalized relative to the expression of RNU6 which served as an internal reference gene [15-18]. The relative amounts of gene expression were analyzed according to the comparative cycle threshold (CT) values method [19] by using the expression of RNU6 for normalization, where $\Delta$ CTwas calculated by subtracting the CT values of RNU6 from the CT values of target miRNAs. The relative expression levels of selected miRNAs were then determined as ratios by dividing the mean of $2 \Delta \mathrm{CT}$ values of cancer group from the mean of $2 \Delta \mathrm{CT}$ values of the healthy controls $[19,20]$.

\section{Statistical analysis}

Statistical analysis was performed using the Statistical Program for Social Sciences (SPSS) software (version 17.0; SPSS Inc., Chicago, USA). All data were presented as mean \pm standard deviation (SD) [21]. The statistical differences of miRNA expression levels between experimental group and control group were analyzed by independent samples $t$ test. $P<0.05$ was considered as statistically significant, $P<0.01$ was considered highly significant. The receiver operating characteristic (ROC) curve was available to evaluate the feasibility of serum miRNA as a biomarker for HCC early diagnosis [22,23]. A stepwise logistic regression model was used to select diagnostic microRNA markers based on the validating dataset. ROC curve and logistic regression 
analysis were performed with MedCalc (version 10.4.7.0.; MedCalc, Mariakerke, Belgium) software.

\section{Results}

\section{RNA-Seq and microRNA screening}

To obtain an expression profile of specific serum miRNAs for HCC, we employed the RNA-seq to compare the miRNA expression levels in HCC serum with that in the healthy controls. As shown in Supplementary Table S2, there was significant variation of serum miRNAs between different samples. From the Solexa sequencing results, 531 miRNAs were detected in 5 mixed HCC samples, while 799 miRNAs were detected in 5 mixed healthy samples, and 417 miRNAs were detected in both samples. It is important that the miRNAs called expressed are really expressed, then a conservative criterion of TPM $\geq 2$ or $\geq 4$ could be used [24]. In this study, we got rid the poorly expressed miRNAs with $\mathrm{TPM}<2$. Among these shared-miRNAs, there were 88 miRNAs were up-regulated and 86 miRNAs were down-regulated in HCC samples, the proportion of miRNAs with fold change larger than 2 is around $41.7 \%$. MiRNAs with 2-folds higher expression in HCC cases than in healthy subjects were selected to search published literatures concerning these genes. MiR-182-5p was reported to be a good prognostic biomarker for glioma progression and patient survival [25]. MiR-363-3p acts as an oncogene in head and neck squamous cell carcinoma [26]. MiR-378a-3p was demonstrated to be an important molecule in various types of human cancer. In the design phase, we referenced the sequencing data of the paper titled "Identification of miRNomes in human liver and hepatocellular carcinoma reveals miR199a/b-3p as therapeutic target for hepatocellular carcinoma" [27], and screened the abundantly expressed microRNAs in liver cancer tissue based on raw data of supplementary information they provided (Supplementary Table S3). MiR-182-5p, miR-363-3p, and miR-378a-3p were over-expressed significantly in tumor tissue than in adjacent tissue, too. These study indicated the candidate miRNAs (miR-182-5p, miR363-3p, and miR-378a-3p) may have potential significant in clinical diagnosis. The differential expression of miRNA between the control and HCC group was revealed by clustering analysis (Supplementary Figure S1).

\section{Validation of $R N U 6$ as an internal reference for quantification}

In order to verify whether RNU6 could serve as an internal reference for quantification of serum miRNAs, we assayed the levels of several reference gene including GADPH, $\beta$-actin and RNU6 by qRT-PCR in 20 serum samples (10 healthy individuals and 10 HCC patients), and the CT values of these reference genes were used to calculate average expression stability value (M) with geNorm software. Gene with the lowest $M$ value has the most stable expression in the test samples [28]. Data analysis with geNorm software showed that RNU6 had the smallest $\mathrm{M}$ value of 0.146 compared with $G A D P H(0.157)$ and $\beta$-actin (0.165), which indicated this small noncoding RNA is stably expressed in serum and is an optimal reference gene for the PCR quantification of other serum miRNAs [29].

\section{Detection of candidate miRNAs profile in HCC samples versus healthy controls}

In order to corroborate the RNA-seq results, we examined the expression levels of the target miRNAs in 52 HCC cases and 25 healthy controls by qRT-PCR. The profiles of miR-182-5p, miR-363$3 p$, and miR-378a-3p in serum samples were analyzed. CT values of miRNAs were normalized with the reference gene RNU6. Similar results were obtained in the qRT-PCR. The selected miRNAs (miR182-5p, miR-363-3p, and miR-378a-3p) showed significantly higher expression in HCC patients than in the healthy controls (fold changes were $3.98,6.78$, and 9.03, respectively) (Figure 1, Supplementary Table S4). The differences were statistically significant $(P<0.01)$. However, we also noticed that although HCC patients had statistically higher 10 average expression of the candidate miRNAs than the healthy controls, a certain degree of overlap appeared between these two groups. This was caused most probably by inter individual variation [22]. ROC curve analysis suggested that serum miR-182-5p, miR-363$3 p$, and miR-378a-3p levels were useful biomarkers for discriminating patients with HCC from the healthy controls. Area under the curve (AUC) was used as an accuracy index for evaluating the diagnostic performance of the selected miRNA panel. AUC of ROC curve analysis at 0.7-0.8 was considered inaccurate, AUC at 0.8-0.9 was considered reasonably accurate while AUC $>0.9$ was considered highly accurate [23]. The ROC curve of circulating miRNAs had an AUC of $0.802,0.845$, and 0.880 , respectively (Figure 2). Among these results, we partly confirmed elevated miRNAs had high accuracy to distinguish HCC from the healthy controls. Worth special noting was that these three miRNAs can also differentiate HCC patients with negative AFP level from the healthy. As a HCC biomarker, serum AFP is currently used in clinical examination. In general, AFP $<20 \mathrm{ng} / \mathrm{ml}$ is considered as normal and AFP $>400 \mathrm{ng} / \mathrm{ml}$ as truly positive of cancer [30]. Compared with the healthy group, the miRNAs expression profile was significantly increased in HCC patients with low AFP (Supplementary Table S5). The qRT-PCR analysis demonstrated that the expression of miR-182-5p was increased approximately 3.01-fold in HCC patients $(\mathrm{n}=16)$ with $\mathrm{AFP}<20 \mathrm{ng} / \mathrm{ml}$ than in normal subjects $(\mathrm{n}=25, P=0.001)$. A similar trend was observed in expression profile of miR-363-3p, which was elevated 10.19-fold in AFP-negative patients $(P=0.002)$. Furthermore, miR-378a-3p was over-expressed 10.68 -fold
A

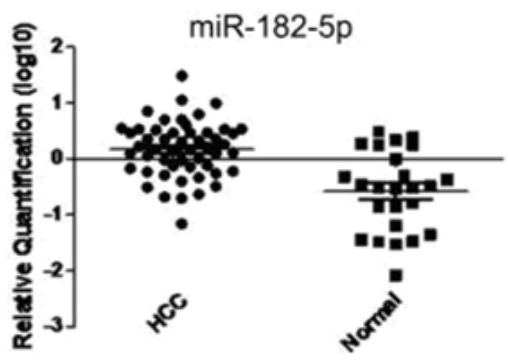

B

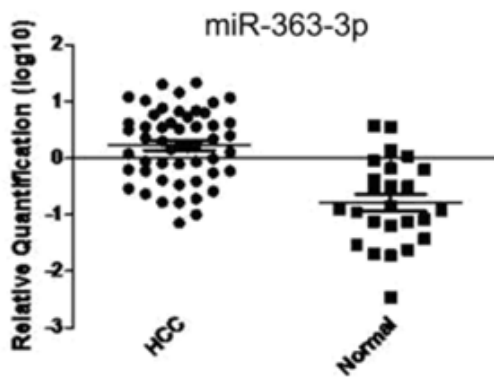

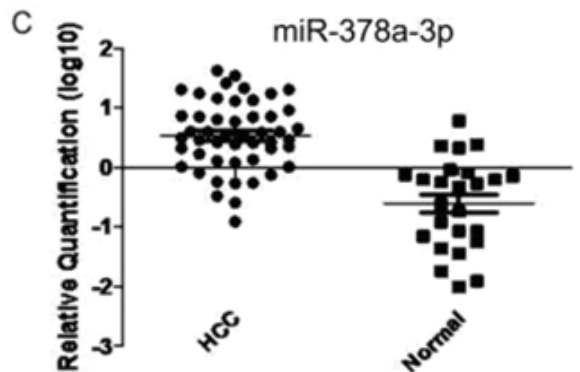

Figure 1: Relative quantification of serum miR-182-5p, miR-363-3p, and miR-378a-3p was presented in HCC patients and controls. MiR-182-5p was expressed 3.98 -folds ( 2.82 vs. $0.71, P<0.001)$, miR-363-3p 6.78 -folds (3.83 vs. $0.57, P<0.001)$, and miR-378a-3p $9.03-$ folds $(7.21$ vs. $0.80, P<0.001)$ compared with the expression levels in the healthy group. 

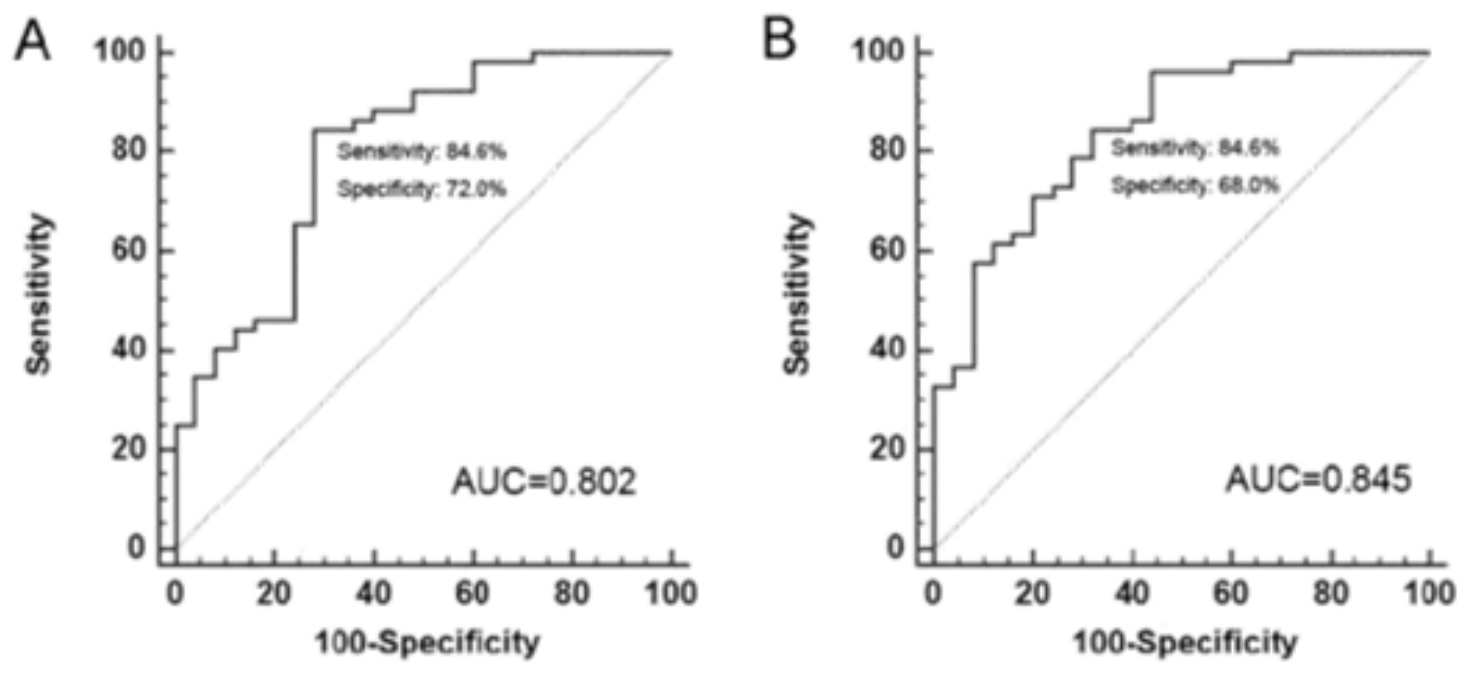

$78 a-3 p$

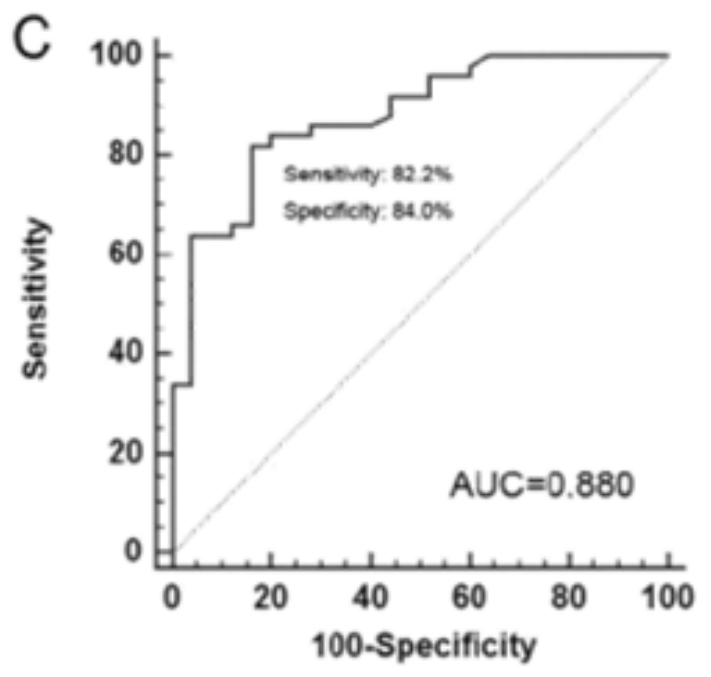

Figure 2: ROC curve analysis of serum miR-182-5p, miR-363-3p, and miR-378a-3p was used to differentiate HCC patients from the healthy controls.

(A) ROC curve of miR-182-5p had an AUC of 0.803 (sensitivity $=84.6 \%$, specificity $=72.0 \%$ );

(B) ROC curve of miR-363-3p yielded an AUC of 0.845 (sensitivity $=84.6 \%$, specificity $=68.0 \%$ ); (C) ROC curve of miR-378a-3p had an AUC of 0.880 (sensitivity=82.2\%, specificity $=84.0 \%$ ).

A

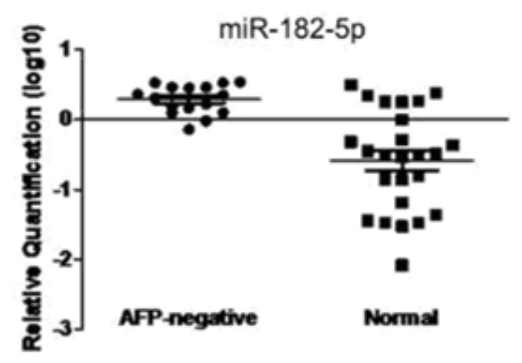

B

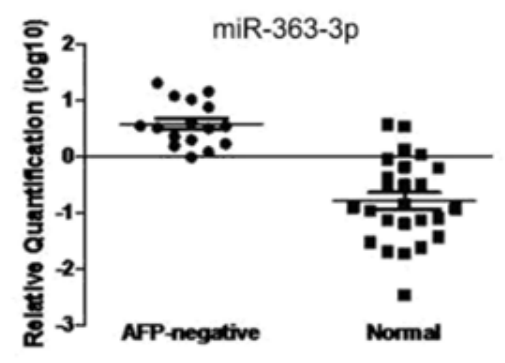

C

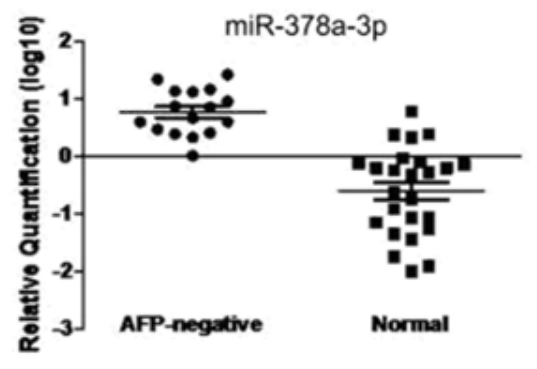

Figure 3: Relative quantification of serum miR-182-5p, miR-363-3p, and miR-378a-3p was presented in AFP-negative HCC patients and controls. MiR-182-5p was expressed 3.01 -folds ( 2.14 vs. $0.71, P=0.001)$, miR-363-3p 10.19-folds (5.76 vs. $0.57, P=0.002)$, and miR-378a-3p 10.68-folds (8.53 vs. $0.80, P=0.001)$ compared with the expression levels in the healthy group.

in AFP-negative group ( $P=0.001)$ (Figure 3$)$. ROC curve analysis of miR-182-5p, miR-363-3p, and miR-378a-3p for discriminating HCC patients with negative AFP level from the healthy controls yielded an
AUC of $0.865,0.930$, and 0.970 , respectively (Figure 4 ). The results illustrated that the tested miRNAs might be used as potentially specific molecular markers for the early diagnosis of HCC subgroup 

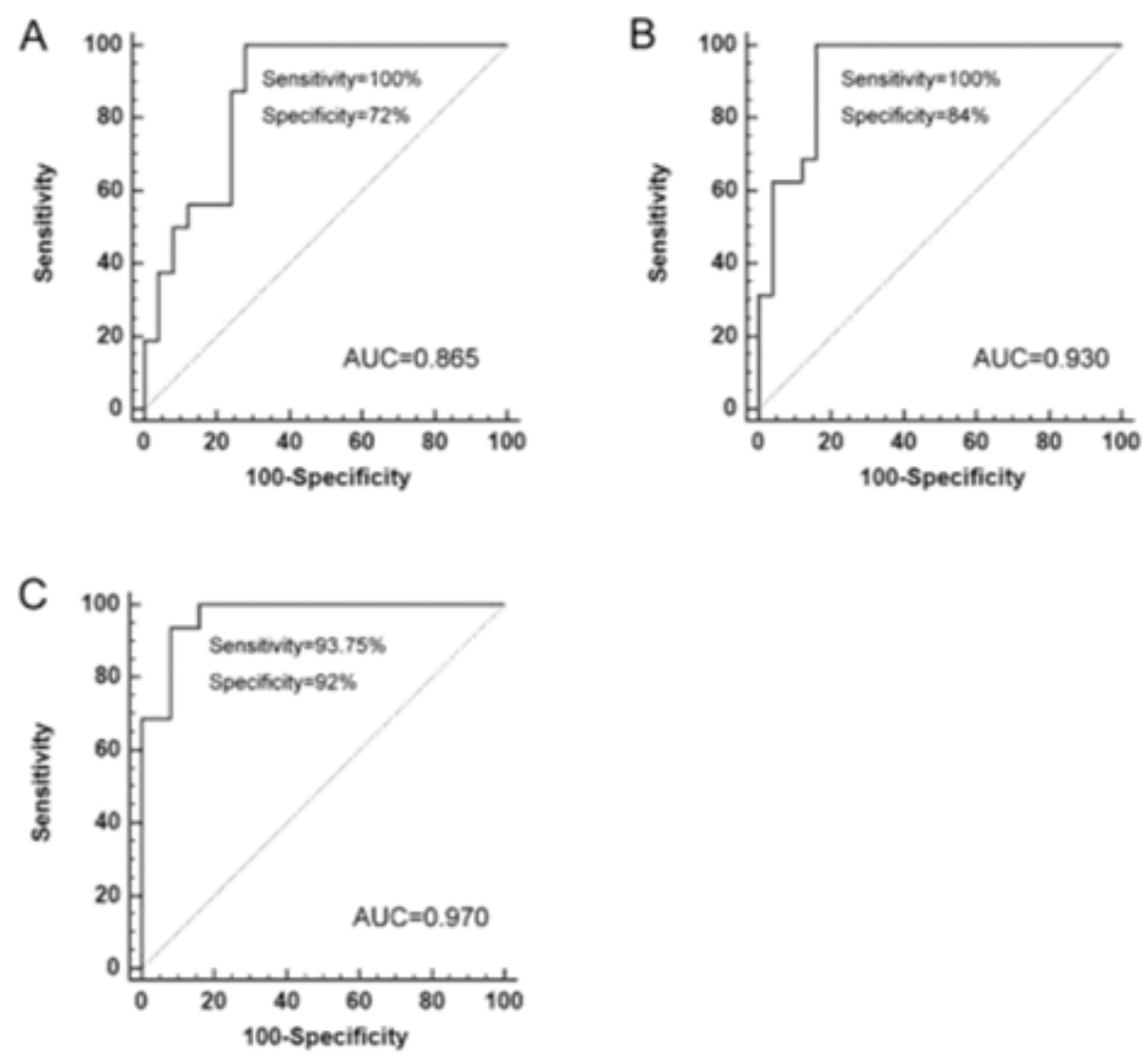

Figure 4: ROC curve analysis of miR-182-5p, miR-363-3p, and miR-378a-3p was used to differentiate AFP-negative HCC patients from the healthy controls. (A) ROC curve of miR-182-5p had an AUC of 0.865 (sensitivity=100\%, specificity=72\%); (B) ROC curve of miR-363-3p had an AUC of 0.930 (sensitivity=100\%, specificity=84\%); (C) ROC curve of miR-378a-3p had an AUC of 0.970 (sensitivity=93.75\%, specificity=92\%).

\begin{tabular}{|c|c|c|c|c|c|c|c|c|}
\hline $\begin{array}{l}\text { MiRNAl } \\
\text { Pathology }\end{array}$ & Age & & AFP & & $\begin{array}{l}\text { HBV DNA } \\
\text { copies }\end{array}$ & & ALT & \\
\hline & $\begin{array}{l}\text { Correlation } \\
\text { coefficient }\end{array}$ & $P$ & $\begin{array}{l}\text { Correlation } \\
\text { coefficient }\end{array}$ & $P$ & $\begin{array}{l}\text { Correlation } \\
\text { coefficient }\end{array}$ & $P$ & $\begin{array}{l}\text { Correlation } \\
\text { coefficient }\end{array}$ & $P$ \\
\hline miR-182-5p & -0.304 & $0.029^{*}$ & -0.456 & $0.001^{* *}$ & -0.453 & $0.001^{* *}$ & 0.046 & 0.76 \\
\hline miR-363-3p & -0.306 & $0.027^{*}$ & -0.276 & $0.048^{*}$ & -0.26 & 0.063 & 0.335 & $0.023^{*}$ \\
\hline miR-378a-3p & -0.061 & 0.669 & -0.447 & $0.001^{* *}$ & -0.431 & $0.001^{* *}$ & 0.312 & $0.04^{*}$ \\
\hline
\end{tabular}

${ }^{*} P<0.05 .{ }^{* *} P<0.01$.

Table 2: The correlation analysis of miRNAs profile to pathology

with negative AFP level. Draw a conclusion, the circulating miRNAs outperformed the serum AFP as tumor biomarkers in detecting HCC cases that could not be detected by the conventional AFP marker.

\section{Establishing the predictive microRNA panel}

To estimate the risk of being diagnosed with HCC, the validating dataset (77 plasma samples) was used to establish a stepwise logistic regression model. All of the tested miRNAs (miR-182-5p, miR363-3p, and miR-378a-3p) were shown to be significant predictors. The predicted probability of being diagnosed with HCC from the logistic regression model based on the three microRNA panel [13], $\operatorname{logit}(\mathrm{p}=\mathrm{HCC})=-0.8959+0.06493 \times \mathrm{miR}-182-5 \mathrm{p}+0.574 \times \mathrm{miR}-363-$ $3 p+0.2861 \times$ miR-378a-3p was used to construct the ROC curve. The diagnostic performance for the established microRNA panel was evaluated by using ROC analysis, and the AUC for the microRNA panel was 0.864 .

\section{Relationship of circulating miRNAs to pathology}

It was reported that some unique miRNA signatures were associated with prognostic factors and disease progression in several cancers [2]. Therefore, we analyzed the correlation between circulating miRNAs expression and clinicopathological parameters. As shown in (Table 2), the correlation analysis showed that the serum miRNAs profile were directly associated with multiple factors, including age, AFP level, hepatitis B virus DNA copies and alanine aminotransferase (ALT), while there's no correlation with aspartate aminotransferase (AST) level. For example, miR-182-5p expression was negatively correlated with age $(P=0.029)$, AFP level $(P=0.001)$ and HBV DNA copies $(P=0.001)$. MiR-363-3p profile was negatively correlated with age $(P=0.027)$, AFP level $(P=0.048)$, while positively correlated with ALT level $(P=0.023)$. 
The expression observed in miR-378a-3p, as similar as the former, was negatively correlated with AFP level $(P=0.001)$ and HBV DNA copies

( $P=0.001)$, while positively correlated with ALT level $(P=0.04)$. Taken together, there are potential relationship between tested miRNAs and pathological factors. What's more, this result might help elucidate the mechanism of hepatocarcinogenesis.

\section{Discussion}

Accumulating evidence indicates that miRNAs play important roles in cancer initiation, propagation, and progression [31]. MiR-182 was localized in 7q31-34 and correlated with gene copy number was reported to be abundantly expressed in human melanoma cell lines and tissue samples [32]. Recent reports showed that miR-182 was involved with the regulation of RGS17 expression by targeting its 3'UTR region in lung adenocarcinomas [33], while another study discovered that upregulation of miR-182 resulted in the down-regulation of metastasis suppressor 1 and increased potential invasive of hepatocellular cell lines [34]. Besides, miR-182 was also considered as a significant prognostic biomarker for glioma progression and patients survival [25]. It suggested that miR-182 might serve as a biomarker for other types of cancer. Limited data was available for miR-363-3p expression in human cancers. Several studies demonstrated that miR 363 acted as an oncogene in head and neck squamous cell carcinoma by directly targeting podoplanin [26], and appeared to be important in HPV pathogenesis [35]. Over-expression of miR-363, on the other hand, reduced tumorigenicity and metastasis in cell invasion assay and liver metastasis in vivo [36]. MiR-363 on Xq26.2 was predicted to correlate with 672 mRNAs [37]. Inhibition of miR-363-3p induced hepatocellular tumorigenesis by promoting G1 to $S$ phase progression, suggested that miR-363-3p might be a negative regulator of Myc and played yet unidentified roles in hepatocarcinogenesis [38]. For future studies about the regulatory mechanism of serum miRNAs in HCC, it will just be an interesting topic. Similarly, miR-378a-3p was not well studied, although a few reports suggested that it might exert different roles in different kinds of cancer pathogenesis. In human cancer, including colorectal cancer [39], non-small cell lung carcinoma [9] and gastric cancer [21], miR-378a was an important molecule. Various expression patterns were observed in circulation and tumor tissue samples [40]. Thus more information about the miRNAs expression pattern and their roles in hepatocarcinogenesis is needed urgently. Above all these miRNAs, miR-363-3p and miR-378a-3p were known as passenger strand of the miRNA duplex and were generally regarded as being degraded in the miRNA biogenesis. Only the miRNA from the guide strand was used in transcription and protein synthesis [41]. Investigations indicated that well-conserved passenger strands, particularly conservative in seed sequences, may contribute to a network of cellular communication and molecular regulation [42]. Up to now, molecular mechanisms and roles of miRNA-3p species in the pathogenesis of cancer remain largely unknown. Possible miR-363-3p and miR-378a-3p binding sites by TargetScan $\backslash$ PicTar $\backslash$ RNA22 $\backslash$ PITA $\backslash \mathrm{miR}$ and software were listed in Supplementary Table S6. Our research provided help to understand potential functions of miRNA-3p species. In conclusion, comparing with the healthy controls, we found that serum miR-182-5p, miR363-3p, and miR-378a-3p were significantly elevated in patients with HCC. All three miRNAs revealed potential diagnostic values for HCC. In addition, these miRNAs helped to diagnose liver cancer with low or negative AFP expression. The diagnostic value, however, was limited by one or more of the following factors: limited number of screened miRNAs as well as small sample size [29]. Nonetheless, our data served as a basis for further research, preferably in large prospective studies before miRNAs could be used as a non-invasive screening tool for HCC in clinical practice.

\section{Acknowledgments}

This study was supported by grants from the Science and Technology Development Projects of Hangzhou (No. 20110733Q04), Natural Science Foundation of Zhejiang Province (LY15C050002 to X.D., 2013KYA170 to S Z.)),Public Technology Research and Social Development Project of Zhejiang Province (No. 2015C37031)

\section{References}

1. Rebecca S, Deepa N, Ahmedin J. (2013) Global cancer statistics. CA Cancer J Clin 63: 11-30.

2. Qi P, Cheng SQ, Wang H, Li N, Chen YF, et al. (2011) Serum microRNAs as biomarkers for hepatocellular carcinoma in Chinese patients with chronic hepatitis B virus infection. PLoS One 6: e28486

3. Shen J, Wang A, Wang Q, Gurvich I, Siegel AB, et al. (2013) Exploration of genome-wide circulating microRNA in hepatocellular carcinoma: MiR-483-5p as a potential biomarker. Cancer Epidemiol Biomarkers Prev 22: 2364-2373.

4. Giannelli G, Fransvea E, Trerotoli P, Beaugrand M, Marinosci F, et al (2007) Clinical validation of combined serological biomarkers for improved hepatocellular carcinoma diagnosis in 961 patients. Clin Chim Acta 383: 147152

5. Chen LT, Xu SD, Xu H, Zhang JF, Ning JF, et al. (2012) MicroRNA-378 is associated with non-small cell lung cancer brain metastasis by promoting cell migration, invasion and tumor angiogenesis. Med Oncol 29: 1673-1680.

6. Murakami Y, Yasuda T, Saigo K, Urashima T, Toyoda H, et al. (2006) Comprehensive analysis of microRNA expression patterns in hepatocellular carcinoma and non-tumorous tissues. Oncogene 25: 2537-2545.

7. Wu XJ, Li Y, Liu D, Zhao LD, Bai B, et al. (2013) miR-27a as an oncogenic microRNA of hepatitis B virus- related hepatocellular carcinoma. Asian Pac Cancer Prev 14: 885-889.

8. Skrzypek K, Tertil M, Golda S, Ciesla M, Weglarczyk K, et al. (2013) Interplay between heme oxygenase- 1 and miR-378 affects non-small cell lung carcinoma growth, vascularization, and metastasis. Antioxid Redox Signal 19: 644-660.

9. Chen X, Ba Y, Ma L, Cai X, Yin Y, et al. (2008) Characterization of microRNAs in serum: a novel class of biomarkers for diagnosis of cancer and other diseases. Cell Res 18: 997-1006.

10. Mitchell PS, Parkin RK, Kroh EM, Fritz BR, Wyman SK, Pogosova EL, Peterson $A$, et al. (2008) Circulating microRNAs as stable blood-based markers for cancer detection. Proc Natl Acad Sci USA, 105: 10513-10518.

11. Li Y, Jiang Z, Xu L, Yao H, Guo J, et al. (2011) Stability analysis of liver cancerrelated microRNAs. Acta Biochim Biophys Sin (Shanghai) 43: 69-78.

12. Agrawal D, Chen T, Irby R, Quackenbush J, Chambers AF, et al. (2002) Osteopontin identified as lead marker of colon cancer progression, using pooled sample expression profiling. J Natl Cancer Inst 94: 513-521.

13. Zhou J, Yu L, Gao X, Hu J, Wang J, et al. (2011) Plasma microRNA panel to diagnose hepatitis B virus-related hepatocellular carcinoma. J Clin Oncol 29 4781-4788.

14. Lin L, Lin Y, Jin Y, Zheng C (2013) Microarray analysis of microRNA expression in liver cancer tissues and normal control. Gene 523: 158-160.

15. Ng EK, Chong WW, Jin H, Lam EK, Shin VY, et al. (2009) Differential expression of microRNAs in plasma of patients with colorectal cancer: a potential marker for colorectal cancer screening. Gut 58: 1375-1381.

16. Thomson JM, Newman M, Parker JS, Morin-Kensicki EM, Wright T, et al. (2006) Extensive post-transcriptional regulation of microRNAs and its implications for cancer. Genes Dev 20: 2202-2207.

17. Yang $\mathrm{H}$, Kong W, He L, Zhao JJ, O'Donnell JD, et al. (2008) MicroRNA expression profiling in human ovarian cancer: miR-214 induces cell survival and cisplatin resistance by targeting PTEN. Cancer Res 68: 425-433.

18. Park NJ, Zhou H, Elashoff D, Henson BS, Kastratovic DA, et al. (2009) Salivary microRNA: discovery, characterization, and clinical utility for oral cancer detection. Clin Cancer Res 15: 5473-5477.

19. Schmittgen TD, Livak KJ (2008) Analyzing real-time PCR data by the comparative $\mathrm{C}(\mathrm{T})$ method. Nat Protoc 3: 1101-1108.

20. Livark K, Schmittgen $T$ (2001) Analysis of relative gene expression data using real-time quantitative PCR and the 2 (-Delta Delta C (T) method. Methods 25 402-408. 
Citation: Zhang S, Zhu L, Ding X, Zhu C, Zheng Z (2016) Over-Expressions of Serum miR-182-5p, miR-363-3p, and miR-378a-3p serve as Biomarkers in Hepatocellular Carcinoma. Mol Biol 5: 154. doi:10.4172/2168-9547.1000154

Page 7 of 7

21. Deng H, Guo Y, Song H, Xiao B, Sun W, et al. (2013) MicroRNA-195 and microRNA-378 mediate tumor growth suppression by epigenetical regulation in gastric cancer. Gene 518: 351-359.

22. Xu J, Wu C, Che X, Wang L, Yu D, Zhang T, Huang L, et al. (2011). Circulating MicroRNAs, miR-21, miR-122, and miR-223, in patients with hepatocellular carcinoma or chronic hepatitis. Mol Carcinog 50: 136-142.

23. Ishii K, Taguchi A, Nakamoto T, Ohtsuka M, Sutthiprapaporn P, et al. (2007) Diagnostic efficacy of alveolar bone loss of the mandible for identifying postmenopausal women with femoral osteoporosis. Dentomaxillofac Radiol 36: 28-33.

24. Wagner GP, Kin K, Lynch VJ (2013) A model based criterion for gene expression calls using RNA-seq data. Theory Biosci 132: 159-164.

25. Jiang L, Mao P, Song L, Wu J, Huang J, et al. (2010) miR-182 as a prognostic marker for glioma progression and patient survival. Am J Pathol 177: 29-38.

26. Sun Q, Zhang J, Cao W, Wang X, Xu Q, et al. (2013) Dysregulated miR-363 affects head and neck cancer invasion and metastasis by targeting podoplanin. Int J Biochem Cell Biol 45: 513-520.

27. Hou J, Lin L, Zhou W, Wang Z, Ding G, et al. (2011) Identification of miRNomes in human liver and hepatocellular carcinoma reveals miR-199a/b-3p as therapeutic target for hepatocellular carcinoma. Cancer Cell 19: 232-243.

28. Vandesompele J, De Preter K, Pattyn F, Poppe B, Van Roy N, et al. (2002) Accurate normalization of real-time quantitative RT-PCR data by geometric averaging of multiple internal control genes. Genome Biol 3: RESEARCH0034.

29. Ahn K, Huh JW, Park SJ, Kim DS, Ha HS, et al. (2008) Selection of internal reference genes for SYBR green qRT-PCR studies of rhesus monkey (Macaca mulatta) tissues. BMC Mol Biol 9: 78.

30. Liu AM, Yao TJ, Wang W, Wong KF, Lee NP, et al. (2012) Circulating miR$15 \mathrm{~b}$ and miR-130b in serum as potential markers for detecting hepatocellular carcinoma: a retrospective cohort study. BMJ Open 2: e000825.

31. Zhu K, Dai Z, Zhou J (2013) Biomarkers for hepatocellular carcinoma: progression in early diagnosis, prognosis, and personalized therapy. Biomark Res 1: 10

32. Segura MF, Hanniford D, Menendez S, Reavie L, Zou X, et al. (2009) Aberrant miR-182 expression promotes melanoma metastasis by repressing FOXO3 and microphthalmia-associated transcription factor. Proc Natl Acad Sci U S A 106: $1814-1819$

33. Sun Y, Fang R, Li C, Li L, Li F, et al. (2010) Hsa-mir-182 suppresses lung tumorigenesis through down regulation of RGS17 expression in vitro. Biochem Biophys Res Commun 396: 501-507.

34. Wang J, Li J, Shen J, Wang C, Yang L, et al. (2012) MicroRNA-182 downregulates metastasis suppressor 1 and contributes to metastasis of hepatocellular carcinoma. BMC Cancer 12: 227.

35. Lajer C, Garnæs E, Friis-Hansen L, Norrild B, Therkildsen MH, et al. (2012) The role of miRNAs in human papilloma virus (HPV)-associated cancers: bridging between HPV-related head and neck cancer and cervical cancer. $\mathrm{Br} \mathrm{J}$ Cancer 106: $1526-1534$

36. Qiao J, Lee S, Paul P, Theiss L, Tiao J, et al. (2013) miR-335 and miR-363 regulation of neuroblastoma tumorigenesis and metastasis. Surgery 154: $226-$

37. Wang L, Oberg AL, Asmann YW, Sicotte H, McDonnell SK, et al. (2009) Genome-wide transcriptional profiling reveals microRNA-correlated genes and biological processes in human lymphoblastoid cell lines. PLoS One 4: e5878.

38. Han H, Sun D, Li W, Shen H, Zhu Y, et al. (2013) A c-Myc-MicroRNA functional feedback loop affects hepatocarcinogenesis. Hepatology 57: 2378-2389.

39. Li H, Dai S, Zhen T, Shi H, Zhang F, et al. (2014) Clinical and biological significance of miR-378a-3p and miR-378a-5p in colorectal cancer. Eur J Cancer 50: 1207-1221.

40. Li W, Xie L, He X, Li J, Tu K, et al. (2008) Diagnostic and prognostic implications of microRNAs in human hepatocellular carcinoma. Int J Cancer 123: 16161622.

41. Zhang J, Wang Y, Zhen P, Luo X, Zhang C, et al. (2013) Genome-wide analysis of miRNA signature differentially expressed in doxorubicin-resistant and parental human hepatocellular carcinoma cell lines. PLoS One 8: e54111.

42. Guo L, Lu Z (2010) The fate of miRNA* strand through evolutionary analysis: implication for degradation as merely carrier strand or potential regulatory molecule? PLoS One 5: e11387. 